\title{
BitView: Using Blockchain Technology to Validate and Diffuse Global Usage Data for Academic Publications
}

\author{
Camillo Lamanna ${ }^{1[0000-0002-8522-354 X]}$ and Manfredi La Manna ${ }^{20000-0001-5542-7114]}$ \\ ${ }^{1}$ University of Sydney, NSW 2006, Australia \\ clam5986@uni.sydney.edu.au \\ ${ }^{2}$ University of St Andrews, St Andrews, UK
}

\begin{abstract}
We suggest that blockchain technology could be used to underpin a validated, reliable, and transparent usage metric for research outputs. Previous attempts to create online usage metrics have been unsuccessful largely because it has been difficult to co-ordinate agreement between all parties on the rules of data collection and the distribution of the workload of data synthesis and dissemination. Blockchain technology can be utilized to bypass this co-ordination problem. We propose the creation of a bibliometric blockchain (called BitView) which forms a decentralized ledger of the online usage of scholarly research outputs. By means of a worked example, we demonstrate how this blockchain could ensure that all parties adhere to the same rules of data collection, and that the workload of data synthesis is distributed equitably. Moreover, we outline how public-private key cryptography could ensure that users' data remains private while librarians, academics, publishers, and research funders retain open access to all the data they require. It is concluded that a usage metric underpinned by blockchain technology may lead to a richer and healthier ecosystem in which publishers and academics are incentivized to widen access to their research.
\end{abstract}

Keywords: Bibliometrics, Blockchain, Usage Factor.

\section{$1 \quad$ Introduction}

In this paper, we suggest that blockchain technology could be used to underpin a validated, reliable, and transparent usage metric for research outputs. The argument proceeds in four steps. In Section 2, we outline the reasons why previous attempts to create usage metrics have been unsuccessful; in particular, we note that is has been difficult to co-ordinate agreement between different stakeholders (ranging from global publishing houses to local institutional repositories) with regard both to data standardization and to the correct distribution of the necessary workload. Collating, cleaning, and disseminating usage data - it has been assumed - is expensive and no single body, understandably, wished to undertake this financial and technical responsibility.

In Section 3, we suggest that blockchain technology could be utilized to bypass the workload distribution problem outlined in the preceding section. By analogy to the most well-known application of blockchain (Bitcoin), we suggest that each usage (i.e. 
online view or download) of digital content increments the 'value' of that object's digital object identifier (DOI) by one. Just as each financial transaction is validated by the nodes on the Bitcoin blockchain, each usage event is validated by agreed standards on the proposed bibliometric blockchain. We maintain that this application of blockchain would be a simple, efficient, and decentralized mechanism by which the 'work' of measuring and validating online usage would be efficiently distributed between stakeholders. Moreover, we outline how public-private key cryptography could be used to ensure that: (i) individual users' online activity remains secure; (ii) there would be free, open access to online usage statistics; (iii) publishers and institutional repositories (PIRs) would have an unencrypted log of the usage of their own digital content; (iv) libraries would be able to measure quickly and easily their usage of different publications.

We conclude that a robust online usage metric, facilitated by blockchain technology, would provide granular information and hitherto hidden insights about the temporal and spatial map of the usage of research outputs. Among other things, this could help assess 'impact' as defined by research assessment exercises. Indeed, a transparent and validated online usage metric may help to restructure the incentives of authors, readers, and PIRs - promoting accessibility and reducing paywalls. This, in turn, may lead to a substantial increase in the percentage of research content that is openly accessible. Thus, the bibliometric blockchain may help create a healthier ecosystem for scholarly communication.

\section{Challenges Facing Journal Metrics}

The Journal Impact Factor (JIF) has become increasingly unpopular in recent years. Many argue that it is overly reductive: there is no substitute for reading a journal in order to ascertain the quality of the material within [1]. Some claim that it is unsound, and that the simple measure may be manipulated by certain editorial practices [2-4]. Others identify it as outdated, and suggest journals be ranked by editorial efficiency and the quality of the review process [5].

With this growing unrest, a burgeoning industry has developed to create new metrics of scholarly impact. The Eigenfactor has been founded upon the principle that not all citations are equal, and uses more involved mathematics in order to weight citations from highly-cited journals above a citation from an academic orphan [6]. CiteScore tweaks the formula of the JIF in order, its creators claim, to make it less vulnerable to calculating journal editors [7]. Altmetrics promise a more holistic approach, incorporating data from Facebook, Twitter and a host of other sources into its score [8].

The JIF and many emerging competitors rely on the premise that a citation (or its social media equivalent: a 'Like' or retweet) is the closest available proxy to the far less tangible quality of being rigorous, original, and relevant. This was understandable in the 1950s when the JIF was created - citations were the only measurable proxy and inevitably therefore also the best; but this is no longer the case [9]. 
In the past two decades, as the new citation-based metrics have multiplied in number, there has also been a growing interest in measuring "usage" - typically understood to mean HTML or PDF downloads of an article [10-12]. The mid-2000s saw a number of attempts to add usage data to the arsenal of metrics available to quantify scholarly communication. In the United States, one group proposed the 'usage impact factor' [13]; in the United Kingdom, another group developed the 'usage factor' [14, $15]$ - however, standardizing the way in which these data were collected across repositories proved challenging. In order to ensure "consistent, credible, and comparable" standards when collating usage data, the non-profit organization Counting Online Usage of Networked Electronic Resources, known as Project COUNTER, was set up and released its first code of practice in 2003 [16]. COUNTER standards ensure that compliant publisher and institutional repositories are consistent in their counting of doubleclicks, web robot hits, federated search activity, and so on [17].

In the UK, in 2011, the final report of the PIRUS project devised the following model for the aggregation and dissemination of journal usage data across publishers and institutional repositories:

a full-text entry is downloaded $\rightarrow$ a log entry is created and passed to a "central clearing house" $(\mathrm{CCH}) \rightarrow \mathrm{CCH}$ filters the log entries by COUNTER rules $\rightarrow \mathrm{CCH}$ disseminates usage statistics [18]

However, there was no willing candidate for the role of the $\mathrm{CCH}$ : "PIRUS proposed the establishment of a global central clearing house, ... [but] the majority of publishers were not yet, largely for economic reasons, ready to implement or participate in such a service" [19]. In response to financial reticence on the part of publishers and to the difficulties of creating a global CCH, PIRUS became IRUS-UK, which functions now as an aggregator of usage data from UK institutional repositories [20]. However, the goal of aggregating global usage data from publishers has yet to be achieved.

This is a clear instance of market failure. On the demand side, there is a demonstrable need for reliable usage data: both from publishers keen to record and publicize the global usage of articles published in their journals, and from research institutions and funding bodies keen to measure the (non-citation-based) impact of publications. On the supply side, views and download data are collected by a multiplicity of individual PIRs and are ready to be harvested on a global scale. We surmise that the root cause of this failure is a misalignment of incentives: commercial publishers are inclined to distrust a mechanism that attaches value to post-prints (an open access entity) at the expense of citations (attached to published articles - a private commodity).

\section{BitView}

We suggest that the problem of a creating a global central clearing house could be bypassed by using blockchain technology. Blockchain is "an open, distributed ledger that can record transactions between two parties efficiently and in a verifiable and permanent way" [21]. The most well-known application of blockchain technology is Bitcoin, 
a decentralized digital currency which uses blockchain to record transactions on a public ledger.

For our proposed implementation, the core intuition is that an online view can be seen as a transfer of one unit of "view-currency" from the viewer to the unique DOI of the viewed material. The privacy of the viewer is maintained using cryptographic techniques to ensure that only the PIR through which the viewer accessed the output has access to their identifying data. The proposed implementation would take the form of a consortium blockchain. In a consortium blockchain, a predefined group of nodes can write on the ledger of transactions, and wider group can read the ledger; in the case of BitView, these predefined nodes would be recognized PIRs, and they alone would be able to submit data to the blockchain, while any online user would have access to read the ledger. This is as opposed to a public blockchain, such as Bitcoin, where any user can become a node which both reads and writes to the blockchain.

(see Table 1)

A worked example may help elucidate the mechanics of the implementation

Table 1. BitView blockchain overview.

\begin{tabular}{|c|c|c|}
\hline Step & Overview & Example \\
\hline 1 & $\begin{array}{l}\text { A viewer accesses an output via a } \\
\text { PIR }\end{array}$ & $\begin{array}{l}\text { Joe Bloggs accesses an article from the Journal of } \\
\text { Bloggometrics via the SciBlog Repository }\end{array}$ \\
\hline 2 & $\begin{array}{l}\text { The PIR logs this access in a } \\
\text { standardized format: (user, insti- } \\
\text { tution, DOI, timestamp, } \\
\text { access_type, download_type) }\end{array}$ & $\begin{array}{l}\text { SciBlog records the following on a local server: } \\
\text { (j.bloggs, Blogg College, } \\
10.1000 / \text { blo00000001, } 1 / 1 / 2019 \\
12: 00: 00 \text {, restricted, PDF) }\end{array}$ \\
\hline 3 & $\begin{array}{l}\text { The PIR keeps a record of all } \\
\text { such accesses in a one hour pe- } \\
\text { riod. If a user is logged-in then } \\
\text { their user credentials are rec- } \\
\text { orded, otherwise their IP address } \\
\text { is recorded. }\end{array}$ & $\begin{array}{l}\text { The local SciBlog record contains one hour of us- } \\
\text { age data: } \\
\text { (j.bloggs, Blogg College, } \\
10.1000 / \text { blo00000001, } 1 / 1 / 2019 \\
12: 00: 00, \text { restricted, PDF) } \\
\ldots \\
(1.1 .128 .192, \text { Unknown, } \\
10.1000 / \text { blog00015, } 1 / 1 / 2019 \\
12: 59: 59, \text { open_access, HTML) }{ }^{1}\end{array}$ \\
\hline 4 & $\begin{array}{l}\text { Every hour, each PIR submits its } \\
\text { transaction record to the block- } \\
\text { chain for scrutiny }\end{array}$ & $\begin{array}{l}\text { SciBlog submits its usage data to the blockchain } \\
\text { along with its public key: } \\
30480241 \text { 00C9 18FA } \\
\text { SciBlog deletes its log on the local server and be- } \\
\text { gins recording the next hour of data }\end{array}$ \\
\hline 5 & $\begin{array}{l}\text { A randomly-selected node col- } \\
\text { lates all the submitted transaction } \\
\text { records and applies the } \\
\text { COUNTER rules }\end{array}$ & $\begin{array}{l}\text { Double-counted entries will be removed, as will } \\
\text { accesses from known web bots }\end{array}$ \\
\hline
\end{tabular}

1 In a real-world application, the user credentials and IP address would be encrypted when submitted to another node. 
A different randomly-selected node cross-checks that the COUNTER rules have been applied correctly - if compliant, the new block is encrypted and added to the chain

A researcher or funding body can search the blockchain to see how a particular output is being accessed

A publisher or institutional repository can search the blockchain to see how its own outputs are being accessed; they can also access data about which users are utilizing their resources

A librarian can search the blockchain to see how much usage a particular journal receives through their institutional access
The SciBlog public key is used to encrypt the user data on its transaction record:

(502B 6772 333C 9F8B, Blogg Col-

lege, $10.1000 / \mathrm{blog} 00000001$,

1/1/2019 12:00:01, restricted,

$\mathrm{PDF}$ )

In this way, there is no user-identifiable data on the blockchain record.

The author of a particular DOI can search the public blockchain record for their article (e.g. $10.1000 /$ blog00000001). This will return both the total validated 'view-count' and, as all accesses are time-stamped, a log of when each use-activity took place.

An employee of SciBlog searches "10.1000/blog" - this returns all entries where the Journal of Bloggometrics has been accessed. Recall that in Step 6, SciBlog's public key was used to encrypt the user's details. SciBlog (and only SciBlog) can now use its private key to decrypt this data, giving:

(j.bloggs, Blogg College,

10.1000/b1000000001, 1/1/2019

12:00:00, restricted, $\mathrm{PDF}$ )

Jack Jones, who works for Bloggs College, searches the public blockchain for "Bloggs College" AND "10.1000/blog"; this returns all entries where a user from Bloggs College has accessed the Journal of Bloggometrics.

The key points from Table 1 are that (i) viewers will maintain their privacy insofar as their identity is secured cryptographically when using online resources; (ii) researchers, funding bodies, publishers, institutional repositories, and librarians can all interrogate the blockchain directly to ascertain the relevant usage data, without needing to go back and forth between different parties (i.e. when librarians collate usage reports from different publishers); (iii) the blockchain automatically cross-checks that COUNTER rules are applied correctly.

As each view increments the "currency" value of the DOI by one, we suggest calling the proposed blockchain 'BitView'.

\subsection{The Immediate Benefits of BitView}

Broadly speaking, the benefits of using blockchain technology to keep a decentralized record of validated usage data can be divided into three categories. 
The first advantage is that BitView would be inexpensive to run. Each step of the blockchain process (collating view transactions; submitting these to other nodes; validating, encrypting and cross-validating the data; and incorporating the finalized block into the blockchain) is automated, and therefore requires no time-consuming human input. ${ }^{2}$ With regard to storage requirements, it is worth noting that the Bitcoin blockchain (which has processed 300 million transactions at the time of writing and records almost 10 years of data) can be stored on a modern smartphone. Therefore, the BitView blockchain will be more accessible to smaller PIRs (which do not have the resources necessary to implement COUNTER). Finally, BitView would be inexpensive to update. Currently, when the COUNTER Code of Practice is updated, this requires a lengthy transition period of up to four years for PIRs to become compliant with the new reporting rules [22]. BitView, conversely, could be updated with a simple single upgrade.

Secondly, the blockchain offers complete transparency. Critics of usage metrics have argued that "validating a journal's usage factor is both technically and feasibly impossible for a journal editor" insofar as they would need to "request the original transaction log file from a publisher and have the ability to extract the relevant data, apply COUNTER's Code of Practice, and perform the appropriate calculations on the data" [23]. Using the proposed system, the community of blockchain nodes ensures that COUNTER's Code of Practice has been adhered to. Whereas COUNTER compliance is currently subject to regular, expensive, time-intensive audit, with BitView, COUNTER compliance would be ensured by the BitView nodes in an automated fashion. It should be recalled, however, that this transparency does not come at the cost of privacy: cryptography can be used to ensure that both individual users and institutions do not have any identifiable data visible on the blockchain.

Thirdly, we suggest that BitView usage data will be more robust. According to recent estimates, the majority of website views come from non-human users: bots, spiders, crawlers [24]. Much of this activity is legitimate and useful: Google's bots ultimately allow us to search the web. However, much of it is not. In addition to benign bots, online repositories are frequently subject to scraping attacks where automated processes attempt to collect all available articles, databases, video resources, and so on [25]. Thus, in order for usage data to be in any way useful, the signal of human users must be extracted from this noise. A global blockchain consortium offers one of the most effective solutions to this problem. A decentralized network of nodes allows for a greater ability to identify irregularities in usage. Each new block will be generated from the collated online usage data submitted by all PIRs in the network in the past hour: therefore, the system will be able to identify in real-time if a particular IP address or a certain online resource is over-represented in the data (i.e. because a new web scraper or bot is active). Currently, with data being collated and sent in to a central clearing

Some readers may be aware that Bitcoin is highly resource-intensive - according to some estimates consuming as much electricity as the country of New Zealand. This is because, being a public blockchain, "miners" are required to perform computationally expensive cryptographic calculations in order to provide "proof-of-work". By using a consortium blockchain model, BitView has no miners nor the requirement to provide proof-of-work, and therefore its energy requirements will be very modest. 
house on a monthly basis, such real-time data analysis is not possible and therefore anomaly detection is cruder. Moreover, the decentralized method of recording online usage will create a virtuous circle in data validation - more nodes allow for greater processing power and greater proportional capture of online activity, this in turn allows for more complete data collection and robust data cleaning as outlined above, which in turn increases the value of joining the blockchain consortium to other PIRs; if they join and become nodes, the circle completes another turn.

\subsection{Some Objections}

First of all, some may be skeptical of adopting technology associated with cryptocurrencies in order to log the usage of scholarly online material. Against this, the utility of blockchains are increasingly being recognized by mainstream businesses and governmental organizations - with applications found from American healthcare to Estonian elections, from Swiss re-insurers to Ghanaian land registries [26-29]. It is foreseeable, therefore, that the popular understanding of blockchain will shift to understand it as a core technology of the online and real world. The BitView blockchain is not a lawless band of extra-legal entities, but something more prosaic: a tightly-regulated network of nodes maintaining a secure ledger of links between viewers and DOIs.

Secondly, it may be argued that using blockchain is an over-engineered solution to the problem of collating usage data. In response to this, we maintain that it is only through using blockchain that three key challenges of recording online scholarly activity can be solved: (i) the identity of users needs to be visible to those whose own repositories are being accessed, but not to others; (ii) PIRs must be able to protect their data while making it publicly available; (iii) PIRs must cross-validate one another's usage data without sharing identifiable data publicly. By encrypting viewer-identifying data, using private-public key cryptography to protect the identity of publishers, and using automated technology to cross-validate the application of COUNTER rules, we submit that the BitView consortium blockchain is an efficient solution to the asymmetrical needs of privacy on the one hand and transparency and accessibility on the other. Finally, some may argue that it will be impossible to ensure that online usage data are sufficiently accurate to be of use. This, of course, is an objection to the overall enterprise of measuring online usage, and not one specific to the use of the blockchain. Arguments for and against this point of view have already been made elsewhere [30-32]. However, it is worth recalling that the BitView blockchain will allow for robust and verifiable filtering of non-human online activity. Moreover, by creating a publicly-interrogable ledger of online views, we posit that interested researchers will use this rich and open-access dataset to devise novel and competing view-metrics, much as has been done already for citation data. For example, supposing that the data show a significant variation in online viewing practices across academic disciplines, it would be possible to generate field-weighted view statistics in order to standardize view data across academic fields. However, this example is somewhat superficial compared to what the blockchain could reveal: interested researchers would have access to a hitherto unavailable detailed global picture of academic view-activity. This could highlight how aca- 
demic interest in certain fields moves across time and space and how information disseminates and dissipates. The 'black' repository of online articles Sci-Hub recently released the map of online downloads from its archive over a six-month period [33]. This map, however, offers only a fraction of the insights available of a dataset that captures COUNTER-compliant global PIR online usage.

\subsection{The Downstream Benefits of BitView}

How would the blockchain of accurate usage data affect the field of scholarly communication? In our view, the most significant benefits of BitView will ultimately depend on the changes in behavior by both authors and readers that it will trigger. Under the current system, the archiving of published articles is largely dependent on individual inclination and the customs of different disciplines. Whereas for a particle physicist placing post-prints on ArXiv is typically a matter of course, in most other disciplines the extent of archiving in institutional repositories is very patchy, with large swathes of published material being available only behind a paywall.

The reasons for the widespread reluctance to post post-prints are easy to identify: once a paper has been accepted for publication, the author rightly feels that the main objective has been achieved. A marker has been placed on the research landscape that will be preserved by the publisher in a form suitable for garnering citations, which, in conjunction with the prestige of the journal, constitute the acknowledged currency of academic recognition and esteem. The incentive to archive a post-print in a repository is rather weak: even assuming that the open access availability of a post-print increases the volume of views, the link from a post-print view to the citation of the published article is tenuous and in many cases impossible because of paywalls.

The incentive to post post-prints would be radically changed if views had a direct impact on the academic value of published research and were not regarded merely as an indirect means to increase citations. This, of course, would require the collection, verification, and diffusion of reliable data on views which can then be summarized in robust metrics. But this is precisely what BitView can provide. Even in countries where there is no centralized impact-based system of research funding (as provided, for example, by Research Excellence Framework in the UK), authors will want to add academic value to their published research by ensuring that data of views of their postprints are validated by internationally-agreed protocols (such as COUNTER). As soon as views count, the counting of views matters. Authors will find that it is in their own interest to archive their post-prints in their institutional repository (IR) so that viewcurrency funds accrue to their own view-account. Moreover, the importance of online views will incentivize IRs to join the blockchain if they have not already done so. It is difficult to underestimate the benefits of a simple recording mechanism (as provided by BitView) whereby for every published article there is at least one freely accessible post-print counterpart. The Holy Grail of universal open access to academic publications has so far eluded the efforts of many individuals and organizations concerned about the inefficient dissemination of knowledge that is inevitably implicit in the proprietary nature of articles published in for-fee access journals. In addition to this, the paywall barriers to the dissemination of knowledge perpetuate research inequalities: the 
best-funded institutions have access to the greatest body of research on which to draw, placing them at an inherent and unfair advantage over the institutions that do not have the same resources. In this way, the status quo is perpetuated and reinforced.

It is easy to see that the availability of BitView will also change viewing behavior. Whereas under the current chaotic and unregulated mechanism for viewing postprints (and published articles) views do not constitute a valid currency for academic recognition, under BitView instead viewers will have the opportunity of checking that their views-currency is well spent. By accessing research material through a BitViewcompliant repository, viewers not only will be assured that the view data are properly recorded and assigned to authors, but they will be also directly responsible for their privacy settings and therefore will have the opportunity to increase the value of their view data. This last point requires some explanation: during the first stage of implementation, we expect BitView to provide accurate, verified, but basic data on views essentially the number of hits per period of time. But we envisage also a second stage in which BitView-compliant repositories will respond to the demand by both authors and academic institutions (University promotion and tenure committees, grant-giving bodies, and especially public and private organizations desirous to use robust non-citation-based impact metrics for the allocation of research funding, etc.) to provide more granular data on views. For example, researchers publishing articles on geographicallyrelevant matters (e.g., malaria in sub-Saharan Africa) may wish to know the location of their viewers, as it would add a completely new dimension of "impact" to the number of citations of their papers. Repositories would then offer a menu of privacy settings to viewers, ranging from (default) total anonymity to location (continent, country, region, city) to personal details (e.g., academic status/location).

Three virtuous circles would propel the diffusion of knowledge: in a BitView world trusted repositories would guarantee transparency as well as safeguarding individual privacy, thereby making views data both reliable and valuable. At the same time concerned authors keen to maximize the 'usage factor' of their work (i.e. maximize the number of views and downloads it receives across platforms) would have a strong incentive to deposit their post-prints in BitView-compliant institutional repositories, and finally responsible viewers would play a role in enriching views-impact data. The endresult of this interaction would be a substantial increase in the availability of openaccess accurate versions (i.e., post-prints) of published, proprietary, articles.

\section{Conclusion}

In conclusion, we argue that this use of blockchain can solve a fundamental problem in creating a transparent network of PIRs - it allows the creation of a ledger of downloads that is simultaneously public (and therefore all nodes in the blockchain can agree that the COUNTER rules have been applied correctly) and private (with the usage statistics of each individual PIR available only to them via their private key). It creates a decentralized record of global data where the (very modest) computational and data storage workload can be equitably distributed across PIRs. Finally, and most importantly, it would allow the development of robust data regarding the usage of material online. 
With validated and accepted criteria for demonstrating that all views are being treated alike, we envisage that it will finally be possible to standardize usage metrics, which will then gain currency in benchmarking research outputs. This will create a publishing ecosystem which incentivizes authors and publishers to widen access to their outputs, encourages readers to share their viewing habits wisely, and allows everybody to understand better the temporal and spatial spread of scholarly communication.

\section{References}

1. Seglen PO (1997) Why the Impact Factor of Journals Should Not Be Used for Evaluating Research. British Medical Journal 314(7079): 498-502

2. The PLoS Medicine Editors (2006) The Impact Factor Game. PLoS Medicine 3(6): e291

3. Arnold DA, Fowler KK (2011) Nefarious Numbers. Notices of the American Mathematical Society 58: 434-437

4. Martin BR (2016) Editors' JIF-Boosting Stratagems - Which are Appropriate and Which Not? Research Policy 45(1) 1-7

5. Vanclay J (2012) Impact Factor: Outdated Artefact or Stepping-Stone to Journal Certification? Scientometrics 32(2): 211-238

6. Bergstrom CT (2007) Eigenfactor: Measuring the Value and Prestige of Scholarly Journals. College and Research Libraries News 68(5) 314-316

7. Zijlstra H, McCullough R (2016) CiteScore: A New Metric to Help You Track Journal Performance and Make Decisions. https://www.elsevier.com/editors-update/story/journal-metrics/citescore-a-new-metric-to-help-you-choose-the-right-journal

8. Trueger NS, Thoma B, Hsu CH, Sullivan S, Peters L, Lin M (2015) The Altmetric Score: A New Measure for Article-Level Dissemination and Impact. Annals of Emergency Medicine 66(5): 549-553

9. Lindsey D (1989) Using Citation Counts as a Measure of Quality in Science: Measuring What's Measurable Rather Than What's Valid. Scientometrics 15(3-4): 189-203

10. Bollen J, Van de Sompel H, Smith JA, Luce R (2005) Toward Alternative Metrics of Journal Impact: A Comparison of Download and Citation Data. Information Processing \& Management 41(6): 1419-1440

11. Kurtz MJ, Bollen J (2012) Usage Bibliometrics. Annual Review of Information Science and Technology 44(1): 1-64

12. Glänzel W, Gorraiz J (2015) Usage Metrics Versus Altmetrics: Confusing Terminology? Scientometrics 102(3): 2161-2164

13. Bollen J, Van de Sompel H (2008) Usage Impact Factor: The Effects of Sample Characteristics on Usage-Based Impact Metrics. Journal of the Association for Information Science and Technology 59(1) 136-149

14. Pesch O (2012) Usage Factor for Journals: A New Measure for Scholarly Impact. The Serials Librarian 63(3-4): 261-268

15. Shepherd P (2013) Altmetrics, PIRUS and Usage Factor. Insights 26(3): 305-310

16. Fleming-May RA, Grogg, JE (2012) Standards, Tools, and Other Products. Library Technology Reports 46(6) 11-16

17. Project COUNTER (2012) The COUNTER Code of Practice for e-Resources: Release 4. https://www.projectcounter.org/wp-content/uploads/2016/01/COPR4.pdf

18. Shepherd P, Needham P (2011) PIRUS2: Final Report. http://www.cranfieldlibrary.cranfield.ac.uk/pirus2/ 
19. MacIntyre R, Alcock J, Needham P, Lambert J (2015) Measuring the usage of repositories via a national standards-based aggregation service: IRUS-UK. In: Schmidt B, Dobreva M (eds) New Avenues for Electronic Publishing in the Age of Infinite Collections and Citizen Science: Scale, Openness and Trust: Proceedings of the 19th International Conference on Electronic Publishing. Amsterdam, Netherlands: IOS Press, 83-92

20. Needham P, Stone G (2012) IRUS-UK: Making Scholarly Statistics Count in UK Repositories, Insights 25(3): 262-266

21. Iansiti M, Lakhani KR (2017) Truth about Blockchain. Harvard Business Review (Jan-Feb): $118-127$

22. Project COUNTER (2017) Transition Timeline. https://www.projectcounter.org/wp-content/uploads/2017/07/transition-timeline-005-1.pdf

23. Davis P (2011) The Journal Usage Factor - Think Locally, Act Locally. http://scholarlykitchen.sspnet.org/2011/09/29/journal-usage-factor-think-locally-act-locally/

24. Zeifman I (2016) Bot Traffic Report 2016. https://www.incapsula.com/blog/bot-traffic-report-2016.html

25. Foster $Z$ (2018), academia.edu, personal communication

26. Orcutt M (2017) Who Will Build the Health-Care Blockchain? MIT Technology Review (September). https://www.technologyreview.com/s/608821/who-will-build-the-healthcare-blockchain/

27. Heller N (2017) Estonia, the Digital Republic. New Yorker (December 18-25). https://www.newyorker.com/magazine/2017/12/18/estonia-the-digital-republic

28. Lerner M (2017) Blockchain Technology Breaks Through. Business Insurance (7 Mar). http://www.businessinsurance.com/article/20170703/NEWS06/912314245/Blockchaintechnology-breaks-through-to-the-insurance-industry

29. Ayemoba A (2017) Africa's First Multinational Blockchain Land Registry to be Launched in Kenya and Ghana. Africa Business Communities (December). https://africabusinesscommunities.com/news/africa $\%$ E2\%80\%99s-first-multinational-blockchain-land-registry-tobe-launched-in-kenya-and-ghana/

30. Luther L (2001) White Paper on Electronic Journal Usage Statistics. Serials Librarian 41(2): 119-48

31. Peters TA (2002) What's the Use? The Value of e-Resource Usage Statistics. New Library World 103(1-2): 39-47

32. McDowell N, Gorman GE (2004) The Relevance of Vendors' Usage Statistics in Academic Library E-Resource Management: A New Zealand Study. Australian Academic \& Research Libraries 35(4): 322-344

33. Bohannon J (2016) Who's Downloading Pirated Papers? Everyone. Science (28 Apr). http://www.sciencemag.org/news/2016/04/whos-downloading-pirated-papers-everyone 